\title{
The influence of flow modification on air and PCM temperatures in an accumulative heat exchanger
}

\author{
${\text { Marcin } \text { Borcuch }^{1 *} \text {, Michat Musiał }}^{1}$, Karol Sztekler ${ }^{1}$, Wojciech Kalawa ${ }^{1}$, Stanisław Gumuła ${ }^{1}$ and Sebastian Stefański ${ }^{1}$ \\ ${ }^{1}$ AGH University of Science and Technology, A. Mickiewicza Av. 30, 30-059 Krakow, Poland
}

\begin{abstract}
The paper presents the influence of flow modification on the operation of an accumulative heat exchanger. This device can be used as a regenerator in ventilation and air supply systems. A heat exchanger uses ceresine (a mixture of paraffins) as a phase change material (PCM). The aim of this research was to determine the effect of flow modification on temperature distribution and pressure drops in the device. The introduction contains a short description of the test stand used, including the accumulative heat exchanger, the guide vanes, and the locations of measurement and control equipment. We found that additional objects limited vortex structures, increased the inside temperature, and dropped the pressure along the heat exchanger. Guidelines for further research are proposed and briefly discussed.
\end{abstract}

\section{Introduction}

The increasing use of energy today has necessitated a search for alternatives in the energy sector. Harsher UE energy politics are commissioning research on new technologies that can lower energy costs, decrease environmental impact and improve energy efficiency. Renewable technologies that have rapidly developed within the last decade, especially those for harnessing wind and solar energy, are proof of their growing popularity. Unfortunately, the application of wind turbines and PV panels strongly depends on weather conditions. It is difficult to operate with high efficiency, and seasonal changes limit the effectiveness and profitability of these technologies. One solution is to store energy produced in off-peak energy consumption periods, and use it later to meet demands. The accumulative heat exchanger constructed at the Department of Thermal and Fluid Flow Machines is a device that allows storage of heat in a PCM. Due to its high latent heat values [1], the device is a suitable solution for heat storage. The application of PCMs in heat exchangers has been studied since the late 1980s [2]. However, the design and development of such devices intended for ventilation and air conditioning systems is relatively new $[3,4]$. Much research is being carried out in an effort to improve the heat transfer coefficient, minimize flow losses, and improve the insulation of whole construction. The geometry proposed in $[5,6]$ has been investigated, but the results obtained showed that the flow arrangement should be modified. The PCM used ceresine as a heat storage material, which is a neutral product formed from the distillation of crude oil. The energy accumulated ranged from $240 \mathrm{~kJ}$ to $880 \mathrm{~kJ}$.

\section{Developing simulation models}

The experimental test stand allows measurement and control of all operating parameters of the accumulative heat exchanger (Fig. 1). An electric air heater is placed at the beginning of the test stand to control temperature and monitor the relative humidity of air entering the system. The accumulative heat exchanger is equipped with 6 thermocouples: two for measuring the temperature of ceresin in the selected cassette; and four for measuring the temperature of air between cassettes. Additionally, two thermocouples measure the temperature of air entering and leaving the heat exchanger. The throttling valve fitted at the heat exchanger exhaust is used to regulate the rate at which air flows into the system. The flowmeter with guide vanes determines the volumetric airflow rate in the heat exchanger. The entire system is driven by a centrifugal fan, located at the end of a duct. The rotational speed of the fan is set precisely by an inverter. Pressure losses $\Delta p$ in the heat exchanger are estimated by measuring static pressure at the inlet and outlet.

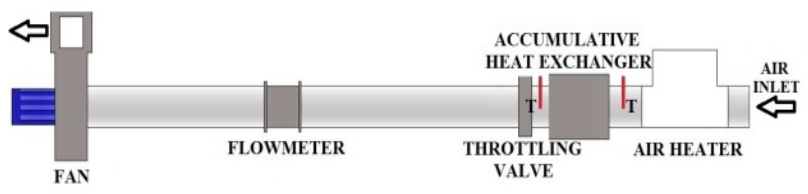

Fig. 1. Experimental stand ( $\mathrm{T}$ - temperature measurement points in the center of the duct).

Two configurations of the accumulative heat exchanger have been examined. At the beginning, measurements were carried out on four cassettes. Each of them was filled with $1.25 \mathrm{~kg}$ of ceresine. In the

Corresponding author: borcuch@agh.edu.pl 
second step, steel guide vanes were prepared and fitted on the edges of the cassettes. These two configurations are shown in Fig. 2 and Fig. 3. The ceresine parameters are presented in Table 1.
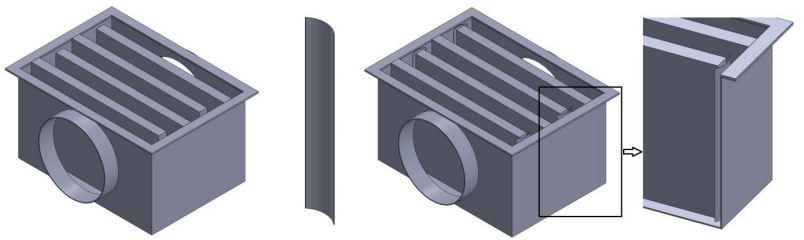

Fig. 2. Two configurations of the accumulative heat exchanger and the location of the guide vanes.
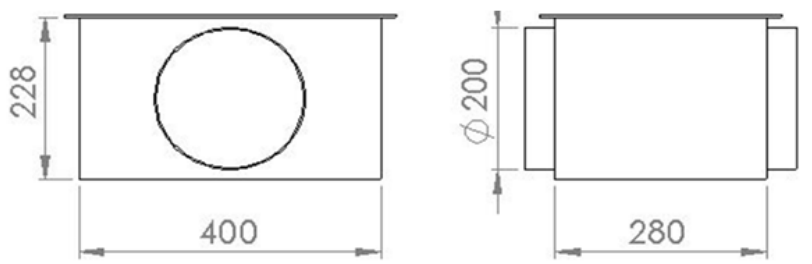

Fig. 3. Dimensions of the device.

Table 1. Ceresine parameters.

\begin{tabular}{|l|c|}
\hline \multicolumn{1}{|c|}{ Parameter } & Value \\
\hline melting temperature range & $61^{\circ} \mathrm{C}$ to $78^{\circ} \mathrm{C}$ \\
\hline density & $0.91 \mathrm{~kg} / \mathrm{m}^{3}$ \\
\hline specific heat capacity & $2.9 \mathrm{~kJ} /(\mathrm{kg}-\mathrm{K})$ \\
\hline thermal conductivity & $0.25 \mathrm{~W} /(\mathrm{m}-\mathrm{K})$ \\
\hline
\end{tabular}

The dimensions of the device are presented in Fig. 3, while the experimental test stand prepared for measurements is presented in Fig. 4. After placing all the thermocouples, the exchanger was covered by a plexiglas sheet and wool-insulated top cover to minimize heat losses.

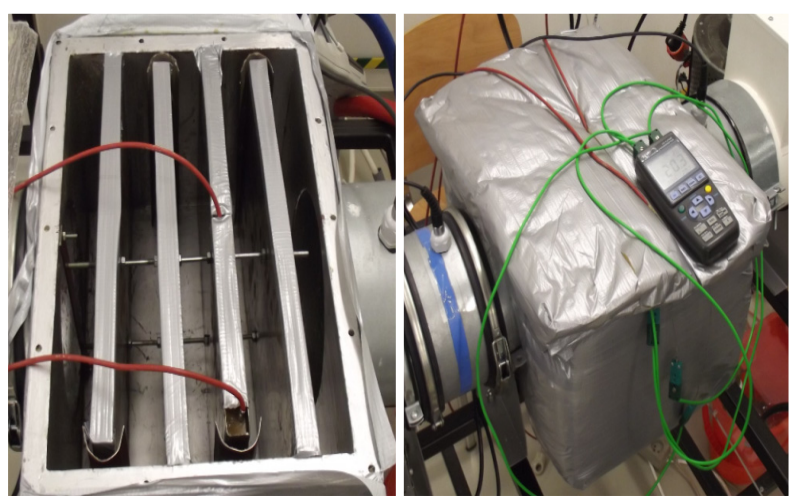

Fig. 4. The accumulative heat exchanger: left - internal view of the exchanger, placement of cassettes, guide vanes, thermocouples to measure the temperature of ceresin; right fully insulated heat exchanger fitted on the experimental stand.

Figure 5 shows a scheme of the analyzed region of the heat exchanger. The arrows indicate the flow directions inside the device. Measuring the temperature at selected points enables estimation of the extent to which the flow modification influences the conditions of heat transfer between the air and the ceresine.

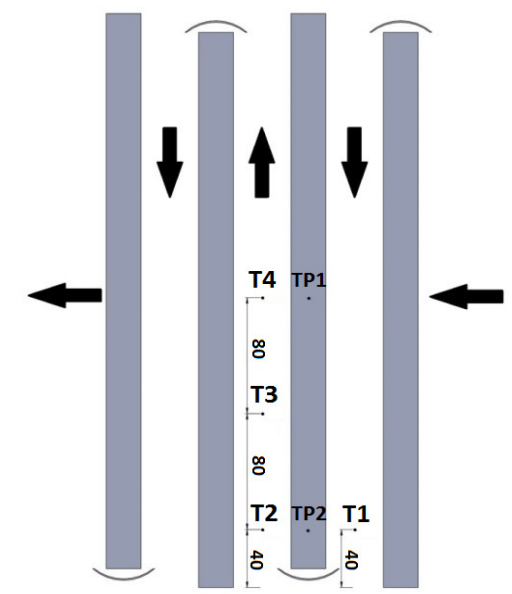

Fig. 5. The air flow direction, guide vanes, the location of thermocouples inside the accumulative heat exchanger.

\section{Experiment and results}

\subsection{Pressure losses}

Experiments were done with and without guide vanes. The influence of the guide vanes can be observed when comparing the thermal and flow parameters of the heat exchanger. Flow modifications introduced additional $\Delta p$ in the heat exchanger, so the entire system required a higher volume flow rate $\dot{V}_{\text {air }}$ maintained by an increased fan rotational speed. This was done to compare the operational parameters of heat exchangers with and without guide vanes under the same flow conditions. The values for static $\Delta p$ in the exchanger are presented in Fig. 6.

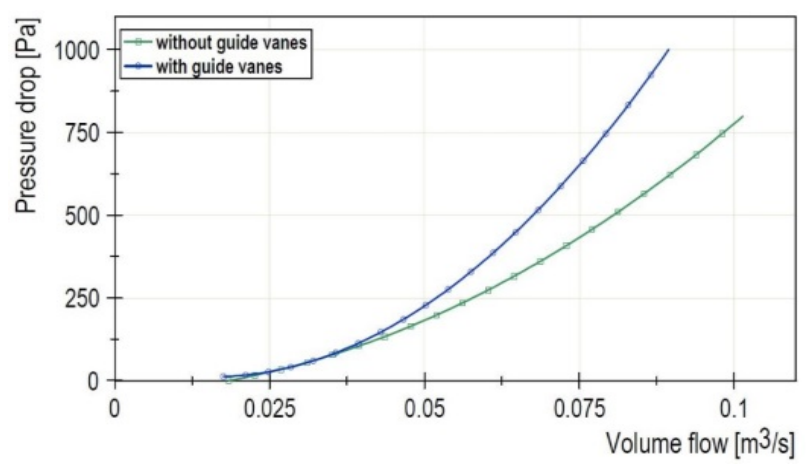

Fig. 6. Measured $\Delta p$ in the accumulative heat exchanger with and without guide vanes.

Higher temperatures of air and ceresin due to the guide vanes was observed, but this came at the price of additional $\Delta p$ (which reached up to $50 \%$ compared to when vanes were not used). 


\subsection{Ceresine and air temperature}

The points of measurement of the air temperature inside the exchanger were located after the second cassette due to stable conditions, and the vortices and disturbances were the same as those after the third cassette. Before the second cassette, there was a meaningful influence of inlet stream, so stable conditions were impossible because of the turbulent nature of the inlet flow. Experiments were done with a $\dot{V}_{\text {air }}$ range of 0.038-0.070 $\mathrm{m}^{3} / \mathrm{s}$, but results are presented only for the two most interesting cases. An increase in air temperature due to the presence of the guide vanes was observed. In particular, temperatures in the vicinity of the guide vanes tended to increase. The increment reached about $7^{\circ} \mathrm{C}$ (Fig. 7 and 8). The temperatures in the modified accumulative heat exchanger appeared to be more greatly varied.

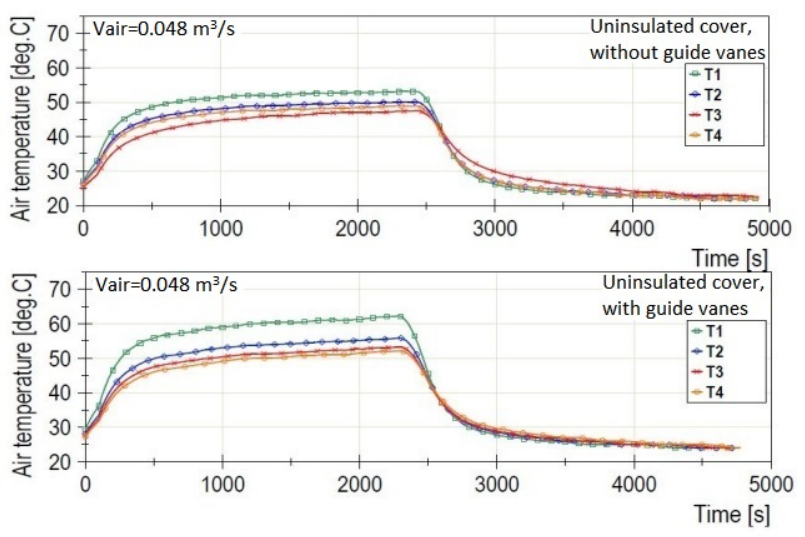

Fig. 7. Air temperature inside the heat exchanger vs time.

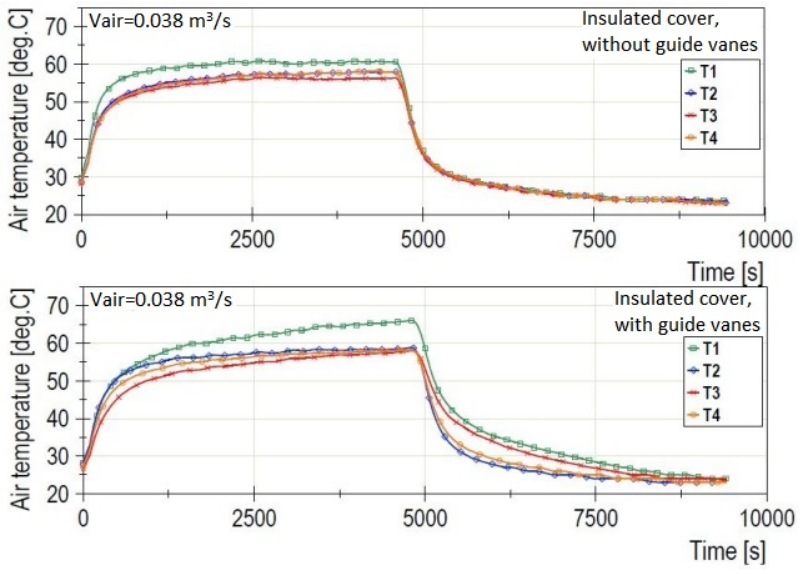

Fig. 8. Air temperature inside the heat exchanger vs time.

Clear differences were observed between T1, T2, T3, and T4, especially during the discharge stage (e.g. Fig. 8 - from 5000 to $7500 \mathrm{~s}$ ). This phenomenon occurred for all flow rates. A noticeable influence caused by the guide vanes was observed in ceresine temperatures as well. The positions of the thermocouples in the cassette allowed measurement of the temperature at two main points: in the middle and on the edge of the cassette. The guide vanes induced faster air flow around the cassettes, so a higher heat transfer coefficient could be expected. This assumption has been confirmed by experiments.
The application of guide vanes caused higher temperatures on the edge of the cassette (Fig. 9). Furthermore, the temperature in the middle of the cassette appeared to be a little lower (Fig. 10), which may have been caused by the absence of vortical structures between modules, and which lead to a smaller flux of heat from the air to the ceresine.

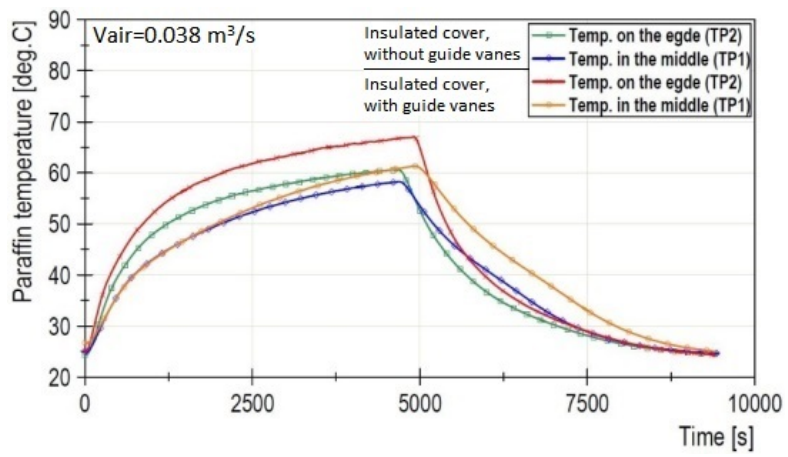

Fig. 9. Heat storage material temperatures inside the second cassette $\left(\dot{V}_{\text {air }}=0.038 \mathrm{~m}^{3} / \mathrm{s}\right)$ vs time.

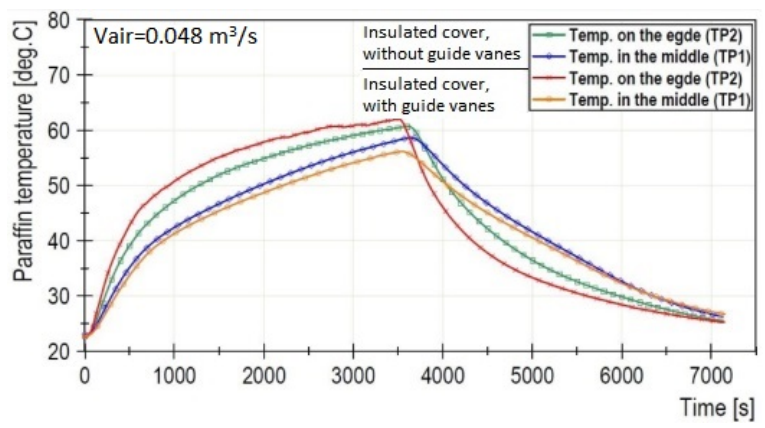

Fig. 10. Heat storage material temperatures inside the second cassette $\left(\dot{V}_{\text {air }}=0.048 \mathrm{~m}^{3} / \mathrm{s}\right)$ vs time.

The temperature measured in the center of the duct is higher than average. Temperature profiles are presented in Fig. 11. The temperature profile at the inlet and outlet pipes of the uninsulated exchanger was measured (Fig. 12).
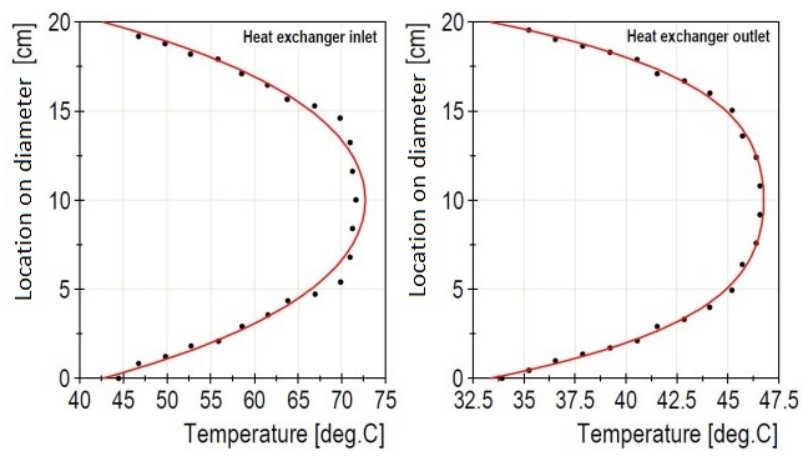

Fig. 11. Temperature profiles at the inlet and outlet of the accumulative heat exchanger.

It can be assumed that average temperatures are: a measured value multiplied by 0.88 at the inlet; and a measured value multiplied by 0.93 at the outlet (Fig. 11). For one case, the values have been recalculated and presented in Fig. 12. One cycle of the exchanger 
operation was divided into two separate parts: the charging stage on the left side; and the discharging stage on the right side. Different temperatures correlate to various air densities:

- density of air at $25^{\circ} \mathrm{C}: \rho_{25}=1.184 \mathrm{~kg} / \mathrm{m}^{3}$;

- density of air at $40^{\circ} \mathrm{C}: \rho_{40}=1.127 \mathrm{~kg} / \mathrm{m}^{3}$.
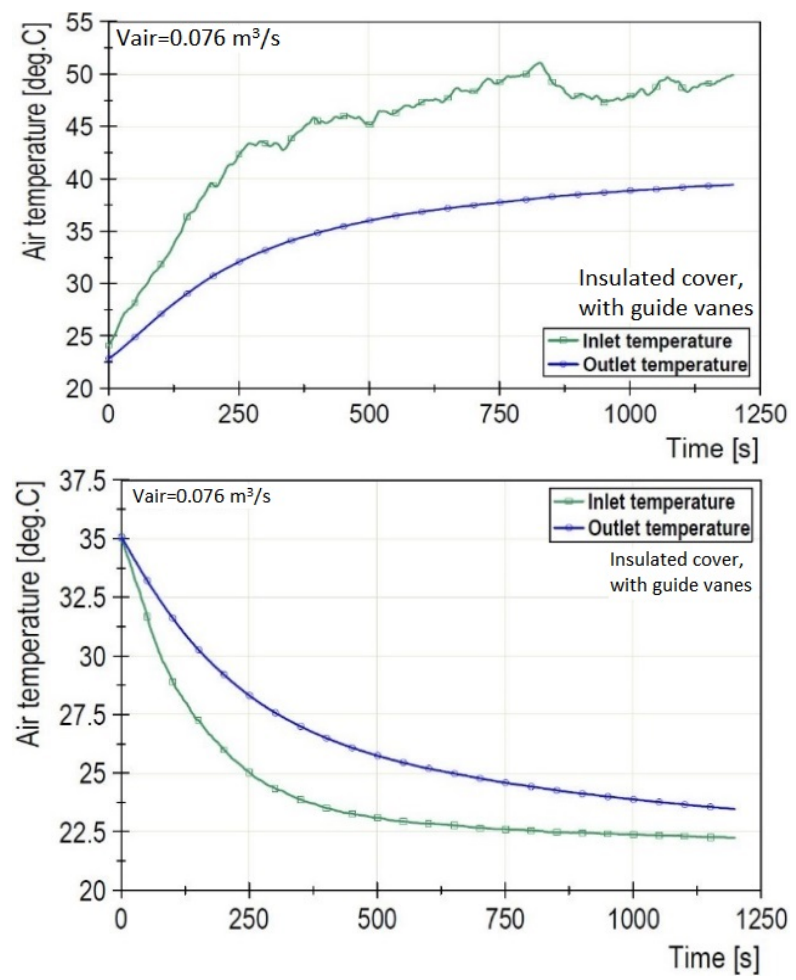

Fig. 12. Average temperatures at inlet and outlet of the exchanger vs time; upper fig. - charging, lower - discharging.

\section{Conclusions}

The application of guide vanes limited the influence of the vortex structures on temperature distribution inside the exchanger. The vanes also increased pressure losses, which induced larger temperatures in flow channels. The compromise between the heat transfer efficiency and flow losses can be balanced by an increase of the rotational speed of the fan. Further research on minimizing the negative impact of guide vanes on flow conditions by modification of their shape and location must be carried out to improve heat exchanger efficiency. For an exchanger with a smaller amount of heat storage modules, the use of cassettes should be considered. The influence of the number of cassettes, their arrangement, and their thickness on accumulated heat are the goals of further research.

\section{Acknowledgment}

The paper was funded from government money Faculty of Energy and Fuels number 11.11.210.216.

\section{References}

1. A. Sharma, V.V. Tyag, C.R. Chen, D. Buddhi, Renew. Sust. Energy Rev. 13318 (2009)

2. M. Okada, J.of Enh. Heat Trasfer 2273 (1995)

3. M. Labat, J. Virgone, D. David, F. Kuznik, Appl. Thermal Engineering 66375 (2014)

4. P. Dolado, A. Lazaro, J. M. Marin, B. Zalba, Renewable Enerrgy 362906 (2011)

5. M. Borcuch, M. Musiał, T. M. Wójcik, Transactions of the Inst. Of Fluid-Flow Mach. 12819 (2015)

6. M. Borcuch, M. Musiał, S. Gumuła, K. Sztekler, K. Wojciechowski, Appl. Thermal Engineering 127 1335 (2017) 